\section{J. Guttmann}

\section{Fundamentals of lung mechanics during mechanical ventilation}

Summary The study of lung mechanics deals with the pressures acting on the respiratory system (lungs and chest wall) and the changes in volume that they produce. In mechanical ventilation, the technical gas delivery system (ventilator, respiratory tubing system, gas humidifier) and the respiratory system of the patient form a connected pneumatic system of high complexity. The respiratory system produces a mechanical impedance to ventilator output. Impedance is composed of an elastic and a non-elastic, i.e., resistive part. The corresponding indices describing respiratory mechanics are compliance and flow resistance. Based on the equation of motion of the respiratory system, the methods of analyz-

PD Dr. rer. nat. Josef Guttmann ( $\varangle$ ) Anaesthesiologische Universitätsklinik Sektion Experimentelle Anaesthesiologie Klinikum der Albert-Ludwigs-Universität Freiburg

Hugstetter Str. 55

D-79106 Freiburg i. Br.

\title{
Grundlagen der Lungenmechanik unter Beatmung
}

ing semistatic compliance and resistance during mechanical ventilation are described. Quantitative analysis of respiratory system mechanics (a) is a prerequisite for the understanding of the complex patient-ventilator interaction, (b) provides important clinical information on pulmonary function and the course of disease, and (c) allows the physician at the bedside to adjust the ventilatory settings to the needs of the individual patient.

Key words Mechanical ventilation respiratory system - lung mechanics equation of motion - compliance resistance - tracheal pressure endotracheal tube

\section{Zusammenfassung Das Gebiet der} Lungenmechanik befaßt sich mit den Drücken, die auf das respiratorische System (Lunge und Thorax) wirken sowie mit den Volumenänderungen, welche diese erzeugen. Bei mechanischer Beatmung bilden das technische Liefersystem für Atemgas (Beatmungsgerät, Atemschlauchsystem, Gasbefeuchter) und das respiratorische System des Patienten ein verbundenes pneumatisches System hoher Komplexität. Das respiratorische System stellt für das Beatmungsgerät eine mechanische Impedanz dar. Die Impedanz setzt sich aus einem elastischen und einem nichtelastischen, d.h. resistiven Teil zusammen. Die entsprechenden Indizes zur Beschreibung der Atemmechanik sind Compliance und Resistance. Auf der Basis der Bewegungsgleichung des respiratorischen Systems wird die Methode zur Analyse der semistatischen Compliance und Resistance unter mechanischer Beatmung beschrieben. Die quantitative Analyse der Atemmechanik des respiratorischen Systems (a) ist die Voraussetzung für das Verständnis der komplexen Wechselwirkung zwischen Patient und Beatmungsgerät, (b) liefert wichtige klinische Information über die Lungenfunktion und den Krankheitsverlauf und (c) ermöglicht es dem Arzt, die Einstellung der Beatmung an die Bedürfnisse des einzelnen Patienten anzupassen.

Schlüsselwörter Mechanische Beatmung - respiratorisches System Lungenmechanik - Bewegungsgleichung - Compliance - Resistance Trachealdruck - Endotrachealtubus 


\section{Einleitung}

Bei Patienten, die wegen schwerer respiratorischer Insuffizienz mechanisch beatmet werden müssen, gewinnt der Kliniker wichtige Information über die Lungenfunktion sowie über Status und Verlauf der pulmonalen Erkrankung durch Analyse und Beurteilung der Atemmechanik [8]. Darüber hinaus ist es ein lange gehegter Wunsch der Intensivmediziner, die Einstellung der mechanischen Beatmung so an die individuelle Atemmechanik des Patienten anzupassen, daß der mechanische Stress für das Lungengewebe und mithin das Risiko für ein pulmonales Trauma minimiert wird $[4,11]$. Gerade in jüngster Zeit wird dieser Problematik verstärkte Aufmerksamkeit gewidmet $[2,9]$. Hinzu kommt, daß die modernste Generation von Beatmungs- und Überwachungsgeräten mit der Darstellung von Druck-, Fluß- und Volumenkurven und von Atemschleifen eine neue Qualität des respiratorischen Monitorings erlaubt, dessen Interpretation das Verständnis der Lungenmechanik voraussetzt. Diese kurzen Vorüberlegungen unterstreichen die Aktualität der Lungenmechanik und rechtfertigen nicht nur die Beschäftigung mit den Grundlagen der Lungenmechanik, sondern machen sie geradezu notwendig. Die Lungenmechanik befaßt sich mit den Drücken, die auf das respiratorische System einwirken und auf die dadurch bedingten Änderungen des pulmonalen Gasvolumens.

Die besondere Situation, mit der wir es bei der mechanischen Beatmung zu tun haben, ist, daß zwei pneumatische Systeme miteinander gekoppelt sind. Das technische Beatmungssystem (Ventilator, Atemschläuche, Befeuchter) ist

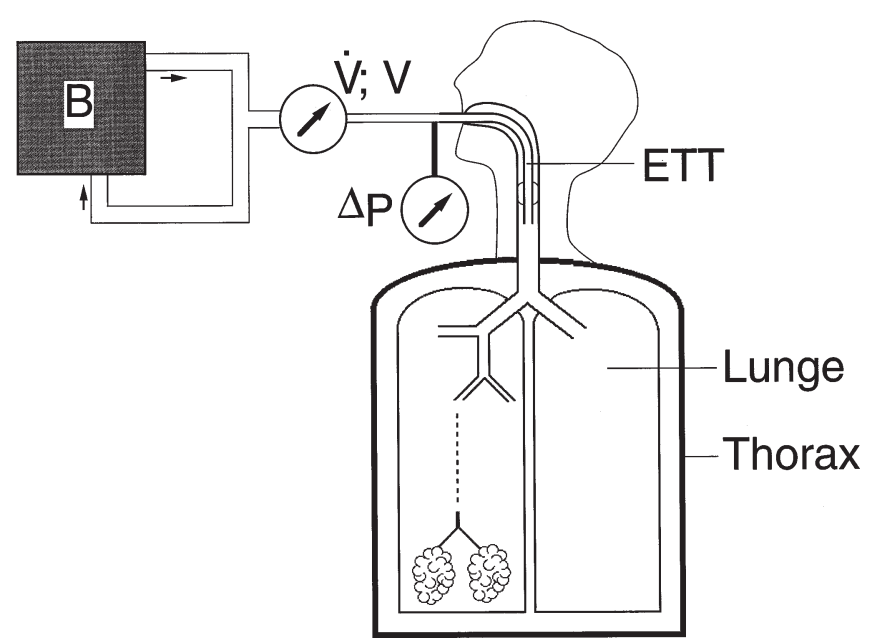

Abb. 1. Schema des respiratorischen Systems (Lunge und Thorax), das durch ein Beatmungsgerät (B) mechanisch beatmet wird. Die Meßstellen für Fluß $(\mathrm{V})$, Volumen $(\mathrm{V})$, und Atemwegsdruck $(\Delta \mathrm{P})$ liegen zwischen Beatmungsgerät und Patient. Der endotracheale Tubus (ETT) ist die pneumatische Verbindung zwischen dem technischen Beatmungssystem und dem biologischen Gasaustauschsystem (mit freundlicher Genehmigung des Fachverlages Schiele \& Schön, Berlin [14]). pneumatisch mit dem biologischen Gasaustauschsystem verbunden. Das pneumatische Verbindungselement ist der endotracheale Tubus (oder die Atemmaske). Beide Teilsysteme stehen in engem und offenem Energieaustausch. Während der Inspirationsphase erhöht der Ventilator den Druck (relativ zu Atmosphäre) in den Atemwegen, um Atemgas in die Lungen zu drücken, während die Exspiration durch den elastischen Retraktionsdruck (elastic recoil pressure) des respiratorischen Systems (Lunge und Thorax) angetrieben wird.

Vom physikalischen Standpunkt aus betrachtet, bildet das respiratorische System des Patienten einen komplexen Widerstand (Impedanz) für das Beatmungssystem. Die Kenntnis der Impedanz des respiratorischen Systems ist eine Grundvoraussetzung für das Verständnis der komplexen Wechselwirkung zwischen Patient und Beatmungsgerät. Das Ziel der vorliegenden Arbeit ist es, die Grundlagen der Lungenmechanik unter Beatmung darzustellen.

\section{Das respiratorische System}

Abb. 1 zeigt schematisch das respiratorische System eines mechanisch beatmeten Patienten. Das respiratorische System besteht aus drei passiven und einem aktiven Subsystem. Im Hinblick auf ihre jeweilige Impedanz umfassen die passiven Subsysteme (a) den respiratorischen Trakt (mit oberen und unteren Atemwegen) und das darin enthaltene Gasvolumen, (b) das Alveolargewebe mit dem darin enthaltenen Gasvolumen und (c) den Thorax (Brustkorb mit assoziierten Muskeln und Zwerchfell). Lunge und Thorax (chest wall) sind mechanisch über den ca. $10 \mu \mathrm{m}$ dicken Pleuralspalt gekoppelt [1] und werden als respiratorisches System im engeren Sinn bezeichnet (gekennzeichnet durch den Index „rs“). Die respiratorischen Muskeln, die in ihrer Gesamtheit oft als „Atempumpe“ bezeichnet werden, sind der aktive Teil des respiratorischen Systems.

\section{Die Bewegungsgleichung des respiratorischen Systems}

Das statische und dynamische Verhalten des respiratorischen Systems wird mathematisch durch die Bewegungsgleichung beschrieben. Diese Gleichung ist in der klinischen Atemphysiologie von zentraler Bedeutung und ist die Basis für alle Verfahren der Atemmechanik-Analyse. Das prinzipielle Verständnis dieser Gleichung sollte daher am Anfang jeder Beschäftigung mit Lungenmechanik stehen.

In der Atemphysiologie werden die mechanischen Eigenschaften des respiratorischen Systems durch einfache physikalische Modelle angenähert. Das einfachste dreidimensionale 


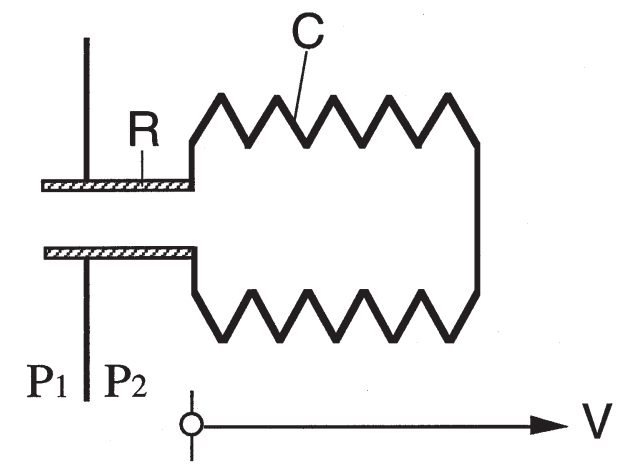

Abb. 2. Physikalisches Modell des respiratorischen Systems bestehend aus einer zylindrischen Röhre mit der Resistance R und einem elastischen Balg mit der Compliance C. Eine Druckdifferenz am Eingang des Modells $\left(\mathrm{P}_{1}-\mathrm{P}_{2}\right)$ bewirkt eine Veränderung seines Gasvolumens V.

Modell des respiratorischen Systems besteht aus einer Röhre und einem elastischen Balg oder Ballon (Abb. 2) [16]. Die Summe aller passiven mechanischen Eigenschaften des respiratorischen Systems werden den beiden Modellkomponenten, Röhre und Balg, zugeordnet. Der Strömungswiderstand oder die Resistance R der Röhre beschreibt die Summe aller Strömungswiderstände des gesamten respiratorischen Systems. Die Volumendehnbarkeit oder Compliance $\mathrm{C}$ des Balges beschreibt die Summe aller elastischen Eigenschaften des respiratorischen Systems. Wird dem System von außen eine Druckdifferenz $\Delta \mathrm{P}$ (transmuraler Druck: $\mathrm{P}_{1}$ minus $\mathrm{P}_{2}$ ) aufgeprägt, so verändert das System sein Volumen (Abb. 2). Damit das System sein Volumen ändert, oder mit anderen Worten, damit Gas in das System fließt, muß der Druck $\Delta \mathrm{P}$ zwei entgegengesetzte Drücke überwinden, (a) den von der Röhre entgegengesetzten ,resistiven“ Druck $\left(\mathrm{P}_{\text {res }}\right)$ und (b) den vom Balg entgegengesetzten ,elastischen“ Druck $\left(\mathrm{P}_{\mathrm{el}}\right)$. Das respiratorische System ist ein Volumen-Druck-System, in dem $P_{e l}$ alle statischen und $\mathrm{P}_{\text {res }}$ alle dynamischen mechanischen Eigenschaften umfaßt.

$\mathrm{P}_{\mathrm{el}}$ hängt vom momentanen Volumen V (über FRC) ab, $\mathrm{P}_{\text {res }}$ hängt von der Änderung des Volumens, d.h. vom Gasfluß V = $\mathrm{dV} / \mathrm{dt}$ ab. Dieser Zusammenhang wird mathematisch durch die Bewegungsgleichung in ihrer allgemeinen Form beschrieben, die besagt, daß der dem System von außen aufgeprägte Druck $\Delta \mathrm{P}$ zu jedem Zeitpunkt gleich der Summe aus $\mathrm{P}_{\mathrm{el}}$ und $\mathrm{P}_{\text {res }}$ ist [16]:

$\Delta \mathrm{P}=\mathrm{P}_{\mathrm{el}}+\mathrm{P}_{\text {res }}=\mathrm{f}_{1}(\mathrm{~V})+\mathrm{f}_{2}(\mathrm{~V})$

Es ist wichtig festzuhalten, daß Gleichung (1) das passive Verhalten des respiratorischen Systems (passive Atemmuskulatur) beschreibt. Um Gleichung (1) in eine für die Praxis handhabbare Form zu bringen, benötigen wir die oben bereits eingeführten Lungenfunktionsindizes Compliance $\mathrm{C}$ (bzw. deren Kehrwert die Elastance) und Resistance R. C verknüpft
Volumen mit Druck, und R verknüpft Fluß mit Druck, d.h. der elastische Druck $\mathrm{P}_{\mathrm{el}}$, der bisweilen auch als Alveolardruck bezeichnet wird, ist $1 / \mathrm{C} \cdot \mathrm{V}$, und der resistive Druck $\mathrm{P}_{\text {res }}$ ist $\mathrm{R} \cdot \mathrm{V}$ :

$\Delta \mathrm{P}=\frac{1}{\mathrm{C}} \cdot \mathrm{V}+\mathrm{R} \cdot \mathrm{V}$

Auf den ersten Blick mag es allzu vereinfachend erscheinen, das komplexe mechanische Verhalten des respiratorischen Systems mit nur zwei Indizes, C und R, zu beschreiben; man kann aber (in Analogie zur elektrischen Netzwerktheorie) ein komplexes mechanisches Volumen-Druck-System, das aus vielen elastischen Elementen und Widerstandselementen besteht, durch jeweils eine äquivalente Compliance und eine äquivalente Resistance beschreiben (RC-Modell). Es konnte gezeigt werden, daß das mechanische Verhalten von normalen Lungen über einen weiten Bereich von Atemfrequenzen auf der Basis dieses Modells zuverlässig vorhergesagt werden kann [20].

Für die Situation der mechanischen Beatmung muß Gleichung (2) erweitert werden: Falls ein positiver endexspiratorischer Druck (PEEP) appliziert wird, entspricht der Druck am Ende der Exspiration ( $\mathrm{V}=0 ; \mathrm{V}=0)$ gerade diesem PEEP beziehungsweise dem (dynamischen) intrinsicPEEP im Falle der unvollständigen Exspiration [12]:

$$
\begin{aligned}
& \Delta \mathrm{P}=\frac{1}{\mathrm{C}} \cdot \mathrm{V}+\mathrm{R} \cdot \mathrm{V}+\mathrm{PEEP} \\
& \Delta \mathrm{P}=\frac{1}{\mathrm{C}} \cdot \mathrm{V}+\mathrm{R} \cdot \mathrm{V}+\mathrm{iPEEP}
\end{aligned}
$$

\section{Indizes der Lungenmechanik}

\section{Compliance}

Die meiste Energie, die dazu benötigt wird, Atemgas in die Lunge zu insufflieren, wird durch das Lungengewebe reversibel (elastisch) gespeichert. Das Lungengewebe umfaßt elastische Strukturen, welche der Lunge die inhärente Eigenschaft geben, sich zu einem geringeren Volumen zusammenzuziehen. Der Thorax verfügt ebenfalls über elastische Eigenschaften. Im Unterschied zur Lunge zeigt der Thorax die Tendenz, nach außen zu ziehen. Die elastischen Eigenschaften von Lunge und Thorax können durch ihre Druck-VolumenKurven beschrieben werden, die unter statischen Bedingungen, d.h. im flußlosen Zustand ( $\mathrm{V}=0)$ gemessen werden. Die Druck-Volumen-Kurve des respiratorischen Systems kann aus den PV-Kurven seiner Komponenten, Lunge und Thorax, 
rekonstruiert werden. Die Compliance des respiratorischen Systems ist definiert als Steigung dieser Volumen-DruckKurve, d.h. als Volumenänderung $\Delta \mathrm{V}$ pro Alveolardruckänderung:

$\mathrm{C}_{\mathrm{rs}}=\frac{\Delta \mathrm{V}}{\Delta \mathrm{P}_{\mathrm{el}}}$

Da Lunge und Thorax in Reihe geschaltet sind, folgt:

$$
\frac{1}{\mathrm{C}_{\mathrm{rs}}}=\frac{1}{\mathrm{C}_{\mathrm{L}}}+\frac{1}{\mathrm{C}_{\mathrm{Th}}}
$$

\section{Resistance}

Um einen Gasfluß durch eine Stromröhre zu erzeugen und aufrecht zu erhalten, ist eine treibende Druckdifferenz zwischen Anfang und Ende des Rohres notwendig. Diese Druckdifferenz überwindet alle durch innere Reibung und Wandreibung bedingten Energieverluste des strömenden Gases.

In der Beatmungstechnik wird üblicherweise der Flow V gemessen. Der Flow, d.h. das pro Zeiteinheit durchgeflossene Volumen, ist an allen Stellen einer Stromröhre gleich, während die Strömungsgeschwindigkeit vom Querschnitt der Stromröhre abhängt. Die wichtige Implikation ist, daß die FlowMessung ortsunabhängig ist. Prinzipiell ist eine Flow-Messung im Respirator gleichwertig mit einer Flow-Messung unmittelbar am Tubusansatzstück (gleiche Meßverfahren vorausgesetzt).
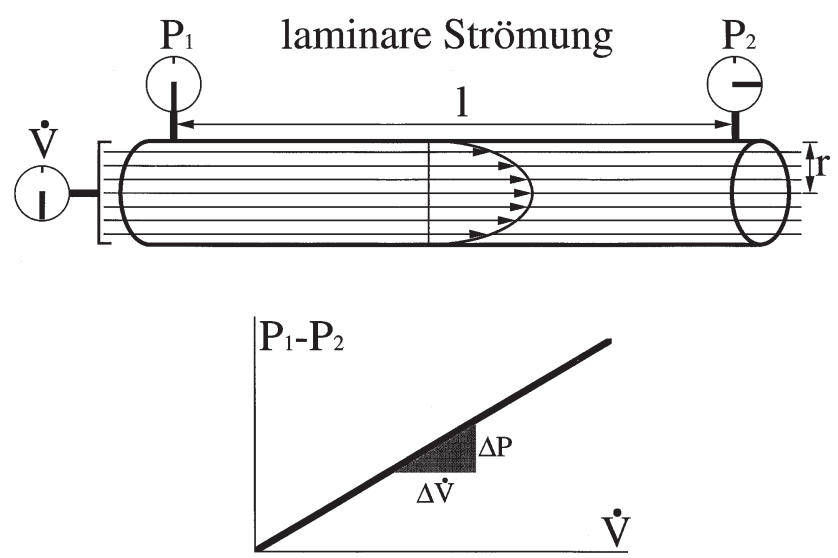

Abb. 3. Zylindrisches Rohrelement mit der Länge 1 und dem Radius $r$. Eine treibende Druckdifferenz $\left(\mathrm{P}_{1}-\mathrm{P}_{2}\right)$ erzeugt einen laminaren Gasfluß V. Die Stromlinien verlaufen parallel zur Rohrachse. Die Geschwindigkeitsverteilung (Strömungsprofil) ist parabelförmig. Das Diagramm zeigt den Zusammenhang zwischen Druckdifferenz und Fluß, der im Fall der laminaren Strömung linear ist. Die Steigung der Geraden entspricht dem Strömungswiderstand, der nach dem Gesetz von HAGENPOISEUILLE (Gleichung (6)) berechnet werden kann.
Abb. 3 zeigt die Situation einer Stromröhre mit kreisförmigem Querschnitt, in dem eine laminare Gasströmung herrscht. Das Verhalten von laminaren Strömungen ist wesentlich durch die innere Reibung bestimmt: es handelt sich dabei um einen gleichmäßig, schichtweise gleitenden Volumenstrom, bei dem die Stromlinien nicht abreißen und weitgehend parallel verlaufen. Mißt man gleichzeitig den Druck $\mathrm{P}_{1}$ am Anfang und $\mathrm{P}_{2}$ am Ende der Stromröhre sowie den Gasfluß V und trägt die Druckdifferenz $\Delta \mathrm{P}_{\text {res }}=\mathrm{P}_{1}-\mathrm{P}_{2}$ über $\mathrm{V}$ auf, so ist die Steigung der Kurve definiert als Strömungswiderstand oder Resistance R:

$\mathrm{R}=\frac{\Delta \mathrm{P}_{\text {res }}}{\mathrm{V}}$

Die Resistance ist also das Verhältnis zwischen treibender Druckdifferenz und erzieltem Fluß. Bei laminarer Strömung ist die Resistance konstant, d.h. unabhängig vom Gasfluß. Das $\Delta \mathrm{P}_{\text {res }} / \mathrm{V}$-Diagramm ist eine Gerade. Für der Strömungswiderstand gilt:

$\mathrm{R}=\frac{\Delta \mathrm{P}_{\mathrm{res}}}{\mathrm{V}}=\frac{8 \cdot \eta \cdot 1}{\pi \cdot \mathrm{r}^{4}}$

Gleichung (6) besagt, daß bei laminarer Rohrströmung in einem kreiszylindrischen Rohr der Strömungswiderstand proportional zur dynamischen Zähigkeit $\eta$ des Strömungsmediums und der Rohrlänge 1 und umgekehrt proportional zur 4. Potenz des Rohrradius $r$ ist. Dieses Gesetz entdeckten der deutsche Wasserbauingenieur HAGEN und der französische Arzt POISEUILLE unabhängig voneinander [5, 21]. Bei voll entwickelter laminarer Strömung ist das Strömungsprofil, d.h. die Geschwindigkeitsverteilung parabelförmig.

Ob eine Strömung laminar bleibt oder turbulent wird, hängt davon ab, ob die an den Strömungsteilchen angreifenden Trägheitskräfte oder die Reibungskräfte (Zähigkeitskräfte) überwiegen. Die von dem englischen Physiker REYNOLDS (1842-1912) zum erstenmal angegebene (und nach ihm benannte) Reynolds-Zahl Re gibt das Verhältnis von Trägheitskräften zu Reibungskräften an (Reibungskräfte werden nach dem NEWTON-Reibungsgesetz, die Trägheitskräfte werden nach dem NEWTON-Grundgesetz der Mechanik abgeleitet; zur Ableitung siehe [5]).

$\operatorname{Re}=\frac{1 \cdot \rho \cdot \mathrm{v}}{\eta}$

mit: 1: charakteristische Länge

$\rho$ : Dichte des strömenden Mediums

v: Strömungsgeschwindigkeit

$\eta$ : dynamische Viskosität

Da bei der mechanischen Beatmung üblicherweise nicht die 
Strömungsgeschwindigkeit, sondern der Gasflow in nahezu kreisrunden Stromröhren gemessen wird, ist folgende Angabe der Reynolds-Zahl handlicher (modifiziert nach [6, 26]):

$\operatorname{Re}=\frac{2 \cdot \rho}{\pi \cdot \eta} \cdot \frac{\mathrm{V}}{\mathrm{r}}=4,23 \cdot \frac{\mathrm{V}}{\mathrm{r}}$

mit: V: Flow in $\mathbf{m L} / \mathbf{s}$

r: Rohrradius in $\mathbf{c m}$

$\rho:$ Dichte des strömenden Mediums: $1.213 * 10^{-3} \mathbf{g} / \mathbf{c m}^{3}$

$\eta$ : dynamische Viskosität (Luft): $1.827 * 10^{-4}$ Poise $\left(1\right.$ Poise $\left.=0.1 \mathrm{Ns} / \mathrm{m}^{2}\right)$

Wichtig ist bei der Verwendung von Gleichung (8), daß die einzelnen Größen in den angegebenen physikalischen Einheiten eingesetzt werden. Dann ergibt sich nach Kürzen aller Einheiten die dimensionslose Reynolds-Zahl.

Die Reynolds-Zahl beschreibt u.a., ob eine Strömung laminar oder turbulent ist. Dominieren die Trägheitskräfte, d.h. die strömenden Teilchen erfahren große Beschleunigungskräfte, so ist die Strömung turbulent, die Reynolds-Zahl ist groß. Dominieren die Reibungskräfte, d.h. die strömenden Teilchen erfahren große Bremskräfte, so ist die Strömung laminar, die Reynolds-Zahl ist klein. Der Umschlag einer Strömung von der laminaren in die turbulente Form erfolgt, wenn die Reynolds-Zahl einen kritischen Wert $\mathrm{Re}_{\text {krit }}$ übersteigt. Als Faustregel kann man bei Gasströmungen für die kritische Reynolds-Zahl einen Wert von 2000 ansetzen:

$\operatorname{Re}=\operatorname{Re}_{\text {krit }}=2000$

Dieser Wert besagt, daß eine Strömung von laminar nach turbulent umschlägt, wenn die Trägheitskräfte die Reibungskräfte um den Faktor 2000 übersteigen. Strömungen mit Reynolds-Zahlen, die kleiner sind als die kritische ReynoldsZahl $\left(\operatorname{Re}<\operatorname{Re}_{\text {krit }}\right)$, sind laminar; für turbulente Strömungen gilt entsprechend: $\operatorname{Re}>\operatorname{Re}_{\text {krit }}$.

Gemäß Gleichung (8) ist Re abhängig vom Flow V und vom Radius $r$ der durchströmten Röhre. Für ein gegebenes Rohr, z.B. einen Endotrachealtubus, hängt damit der Umschlag von laminar nach turbulent von $\mathrm{V}$ ab. Setzt man Gleichung (9) in Gleichung (8) ein und löst nach V auf, so entspricht der berechnete Flow dem kritischen Flow $V_{\text {krit }}$, oberhalb dessen die Strömung turbulent wird.

$\mathrm{V}=\mathrm{V}_{\mathrm{krit}}=\frac{\mathrm{Re}_{\mathrm{krit}}}{4,23} \cdot \mathrm{r}=472,8 \cdot \mathrm{r}$

Führt man diese einfache Rechnung beispielsweise für alle gängigen Trachealtuben durch, so gelangt man zu dem in Abb. 4 gezeigten Zusammenhang zwischen dem kritischen Fluß $V_{\text {krit }}$ und dem Tubusdurchmesser. Das Diagramm in Abb. 4 zeigt die kritischen Flüsse für pädiatrische Endo-

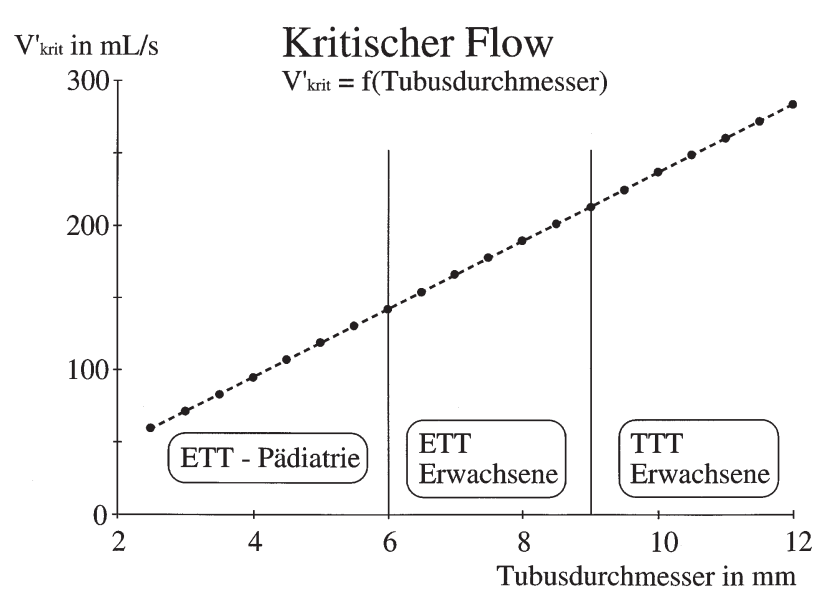

Abb. 4. Kritischer Fluß, bei dessen Überschreiten die Strömung von laminar nach turbulent umschlägt, für alle gängigen Größen von Endotrachealtuben (ETT) und Tracheotomietuben (TTT).

trachealtuben (ETT) von 2,5 mm Innendurchmesser bis zu Tracheotomietuben (TTT) von $12 \mathrm{~mm}$ Innendurchmesser. Wie Abb. 4 zeigt, sind die kritischen Flüsse für Endotrachealtuben relativ gering, d.h. für die bei tracheal intubierten Patienten vorkommenden Flüsse ist der Fluß im Tubus praktisch immer turbulent.

Bei turbulenter Strömung ist der Zusammenhang zwischen Druckdifferenz oder Druckabfall über einem Rohrsegment und dem Flow nichtlinear und hängt in komplizierter Weise von der Rohrgeometrie, vom Staudruck, von der Strömungsgeschwindigkeit und von der Wandrauhigkeit ab [5].

Die nichtlineare Beziehung zwischen Druckabfall und Fluß (Abb. 5) kann mathematisch durch eine Potenzfunktion ausgedrückt werden. In der Atemphysiologie wird die ROHRER-

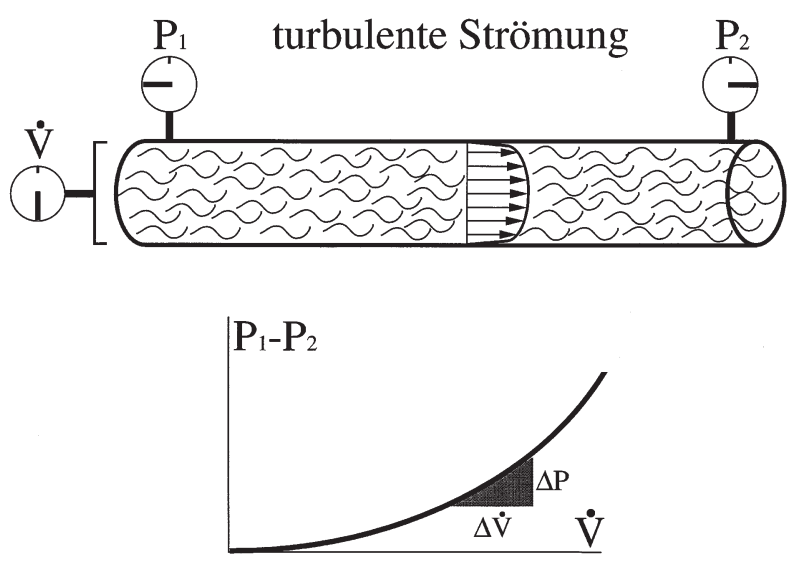

Abb. 5. Zylindrisches Rohrelement mit turbulentem Gasfluß V. Die Geschwindigkeitsverteilung ist flacher als bei laminarer Strömung. Der Zusammenhang zwischen Druckdifferenz und Fluß ist nichtlinear und wird in der Atemphysiologie meist nach der Gleichung von ROHRER (Gleichung (11)) berechnet. 
Gleichung [23] häufig verwendet, die sich lediglich an der mathematischen Beschreibung des nichtlinearen Druck-FlußZusammenhanges orientiert [21]:

$\mathrm{R}=\frac{\Delta \mathrm{P}_{\mathrm{res}}}{\mathrm{V}}=\frac{\left(\mathrm{k}_{1} \cdot \mathrm{V}+\mathrm{k}_{2} \cdot \mathrm{V}^{2}\right)}{\mathrm{V}}=\mathrm{k}_{1}+\mathrm{k}_{2} \cdot \mathrm{V}$

wobei $\mathrm{k}_{1}$ und $\mathrm{k}_{2}$ die empirisch bestimmten Rohrer-Koeffizienten sind.

\section{Konzept der Trachealdruckberechnung}

Gleichung (11) ist ebenfalls dazu geeignet, den flußabhängigen Druckabfall über dem endotrachealen Tubus zu beschreiben. Die Koeffizienten $\mathrm{k}_{1}$ und $\mathrm{k}_{2}$ wurden für alle in der pädiatrischen [15] und in der Erwachsenen [13]-Beatmungstherapie gängigen Tubusgrößen bestimmt. Kennt man diese Tubus-Koeffizienten, so kann man zu jedem momentanten Fluß V den Druckabfall über dem Tubus gemäß Gleichung (11) sehr einfach berechnen. Wie Abb. 1 zeigt, entspricht dieser Druckabfall aber gerade dem Unterschied zwischen dem außen am Tubus herrschenden Atemwegsdruck $\left(\mathrm{P}_{\text {aw }}\right)$ und dem Trachealdruck $\left(\mathrm{P}_{\text {trach }}\right)$. Da der Trachealdruck der für die mechanische Belastung der Lunge entscheidende Druck ist, ist dessen Kenntnis besonders wünschenswert. Da der intratracheale Druck in der klinischen Praxis - zumindest bisher nicht gemessen werden kann, ist dessen kontinuierliche Berechnung eine gute und praktikable Lösung $[13,15]$. Der Trachealdruck ergibt sich aus der Differenz, Atemwegsdruck minus Druckabfall über dem Tubus $\Delta \mathrm{P}_{\mathrm{ETT}}$ :

$\mathrm{P}_{\text {trach }}=\mathrm{P}_{\mathrm{aw}}-\Delta \mathrm{P}_{\mathrm{ETT}}$

Gemäß Gleichung (11) kann $\Delta \mathrm{P}_{\text {ETT }}$ mit den Tubus-Koeffizienten und dem kontinuierlich gemessenen Fluß berechnet werden:

$\mathrm{P}_{\text {trach }}=\mathrm{P}_{\mathrm{aw}}-\left(\mathrm{k}_{1} \cdot \mathrm{V}+\mathrm{k}_{2} \cdot \mathrm{V}^{2}\right)$

Der Fehler der Berechnung von $\mathrm{P}_{\text {trach }}$ liegt unter 1 mbar.

\section{Atemmechanik-Analyse unter Beatmung: semistatische Compliance und Resistance}

Die in der Klinik am häufigsten angewendeten Methoden zur Bestimmung der Atemmechanik sind die sog. ,semistatischen" Methoden, welche statische $(\mathrm{V}=0)$ und dynamische Bedingungen $(\mathrm{V} \neq 0)$ innerhalb eines Atemzyklus kombinieren. Die semistatische Compliance $\left(\mathrm{C}_{\mathrm{sST}}\right)$ und Resistance
$\left(\mathrm{R}_{\mathrm{SST}}\right)$ werden bestimmt mittels eines Null-Fluß-Manövers am Ende der Inspiration (endinspiratorische Pause; EIP). Wie oben gezeigt, beinhaltet die Bewegungsgleichung (2) mindestens zwei unbekannte Größen, Compliance und Resistance, während Druck, Fluß und Volumen gemessene Variablen darstellen. Da es bekanntlich nicht möglich ist, zwei Unbekannte aus einer Gleichung zu bestimmen, entfernt man eine der beiden Unbekannten mit einem „Trick“: man setzt die Flußrate künstlich auf Null durch Einführen von Null-Fluß-Manövern in das Atemmuster. Immer wenn die Flußrate Null ist, verschwindet die resistive Komponente des Atemwegsdruckes $(\mathrm{V} \cdot \mathrm{R})$, und der Atemwegsdruck ist gleich dem Alveolardruck. Auf diese Weise ermöglicht das EIP-Manöver die Trennung zwischen der dynamischen Druckkomponenten, die notwendig ist zur Überwindung des Strömungswiderstandes der Atemwege (und des endotrachealen Tubus) und der statischen Druckkomponenten, die notwendig ist, den elastischen Druck (elastic recoil pressure) zu überwinden [18].

Bei einer EIP ist der Gasfluß Null. Bei vollständiger Exspiration, d.h. wenn das Tidalvolumen vollständig ausgeatmet ist, ist der endexspiratorische Fluß ebenfalls Null. Folglich sind Atemwegsdruck und Alveolardruck an zwei Stellen innerhalb des Atemzuges identisch: am Ende der Inspiration (Index 'Ie') und am Ende der Exspiration (Index 'Ee'). Aus der Bewegungsgleichung folgt:

$$
\begin{aligned}
& \mathrm{P}_{\text {awle }}=\mathrm{P}_{\text {alvIe }}=\frac{\mathrm{V}_{\mathrm{T}}}{\mathrm{C}_{\mathrm{sST}}}+\text { PEEP } \\
& \mathrm{P}_{\text {awEe }}=\mathrm{P}_{\text {alvEe }}=\text { PEEP }
\end{aligned}
$$

Subtrahiert man Gleichung (15) von (14), ergibt sich die Differenz der Alveolardrücke zwischen den beiden Punkten mit Null-Fluß, was die Berechnung der Zwei-Punkt-Gleichung für die semistatische Compliance erlaubt:

$\mathrm{C}_{\mathrm{sST}}=\frac{\mathrm{V}_{\mathrm{T}}}{\mathrm{P}_{\mathrm{awIe}}-\mathrm{PEEP}}$

Eine ähnliche Ableitung ist in [3] beschrieben. Auf diese Weise wird $\mathrm{C}_{\mathrm{sST}}$ bestimmt als Verhältnis von exspiriertem Volumen zu Alveolardruckdifferenz zwischen EIP und dem Ende der Exspiration [10, 25].

Bei vielen Patienten, die wegen einer schweren respiratorischen Insuffizienz mechanisch beatmet werden müssen, sollte die Atemfrequenz erhöht werden, um eine adäquate $\mathrm{CO}_{2}$-Elimination zu gewährleisten. Dies führt zu einer Reduktion der Exspirationszeit bis hin zur unvollständigen Exspiration, wobei das nicht ausgeatmete, also retinierte Gas den mittleren Alveolardruck über dessen Gleichgewichtswert erhöht. Folglich ist der endexspiratorische Alveolardruck höher als der von außen applizierte PEEP, ein Phänomen, das als intrinsic- oder autoPEEP bezeichnet wird [22, 24]. Die Größe des iPEEP 
kann entweder mittels eines endexspiratorischen „Hold (Pause)-Manövers“ bestimmt werden [22], oder aus dem Anstieg des Atemwegsdruckes unmittelbar vor Einsetzen des inspiratorischen Flußes vom nachfolgenden Atemzug [24]:

$\mathrm{P}_{\mathrm{awEe}}=\mathrm{P}_{\mathrm{alvEe}}=\mathrm{iPEEP}$

Für die semistatische Compliance, $\mathrm{C}_{\text {sST.IP }}$, die für den iPEEP korrigiert ist, kann die Zwei-Punkt-Gleichung abgeleitet werden aus der Differenz der Alveolardrücke $\mathrm{P}_{\text {alvIe }}-\mathrm{P}_{\text {alvEe }}(\mathrm{Gl}$. (14) - Gl. (17)):

$\mathrm{C}_{\text {sST,IP }}=\frac{\mathrm{V}_{\mathrm{T}}}{\mathrm{P}_{\text {awIe }}-\text { iPEEP }}$

Rossi et al. haben die Wichtigkeit des iPEEP für die Berechnung der Compliance des respiratorischen Systems sehr überzeugend nachgewiesen: Im Falle unvollständiger Exspiration würde die semistatische Compliance ganz erheblich unterschätzt werden (um bis zu $35 \%$ ), wenn der eingestellte PEEP anstelle des endexspiratorischen Alveolardruckes (iPEEP) zugrundegelegt wird [24]. Ein ganz offensichtlicher Vorteil der EIP-Methode ist ihre rasche und sichere Durchführbarkeit mit den meisten modernen Beatmungsgeräten [7].

Die Bestimmung der semistatischen Resistance $\left(\mathrm{R}_{\mathrm{sST}}\right)$ basiert auf dem Prinzip, das erstmals durch von Neergaard und Wirz beschrieben wurde. Diese Autoren haben den Strömungswiderstand von spontan atmenden Personen aus dem Drucksprung bestimmt, der unmittelbar auf eine kurzzeitige Unterbrechung des Gasflußes folgt [19]. Dieses Prinzip ist von Milic-Emili auf das EIP-Manöver bei mechanischer Beatmung angewendet worden [17]. Der Atemwegsdruck unmittelbar vor (Index: 'v') und unmittelbar nach (Index: 'n') einer EIP-Okklusion läßt sich entsprechend der Bewegungsgleichung folgendermaßen beschreiben:

$$
\begin{aligned}
& \mathrm{P}_{\mathrm{awv}}=\mathrm{P}_{\mathrm{aw}, \max }=\frac{\mathrm{V}_{\mathrm{v}}}{\mathrm{C}}+\mathrm{V}_{\mathrm{Ie}} \cdot \mathrm{R}_{\mathrm{sST}} \\
& \mathrm{P}_{\text {awn }}=\mathrm{P}_{1}=\frac{\mathrm{V}_{\mathrm{n}}}{\mathrm{C}}
\end{aligned}
$$

Da zwischen den beiden Situationen ' $v$ ' and ' $n$ ', welche innerhalb weniger Millisekunden aufeinander folgen, keine Volumenänderung stattfindet, folgt: $\mathrm{V}_{\mathrm{v}}=\mathrm{V}_{\mathrm{n}}=\mathrm{V}_{\mathrm{T}}$. Subtrahiert man Gleichung (20) von (19), erhält man den resistiven Druckabfall $\left(\mathrm{P}_{\mathrm{awv}}-\mathrm{P}_{\mathrm{awn}}\right)$ und damit die Zwei-Punkt-Gleichung der semistatischen Resistance:

$\mathrm{R}_{\mathrm{sST}}=\frac{\mathrm{P}_{\mathrm{aw}, \max }-\mathrm{P}_{1}}{\mathrm{~V}_{\mathrm{Ie}}}$

$\mathrm{P}_{\mathrm{aw}, \mathrm{max}}$ ist der inspiratorische Spitzendruck und $\mathrm{P}_{1}$ ist der Atemwegsdruck, der sich unmittelbar nach der Atemwegsokklusion einstellt (Abb. 6). Es ist wichtig festzuhalten, daß $R_{\mathrm{sST}}$ den Tubuswiderstand beinhaltet. Berechnet man gemäß Gleichung (13) anstelle von $\mathrm{P}_{\text {aw,max }}$ den maximalen Trachealdruck $\mathrm{P}_{\text {trach,max }}$, so kann der Tubuswiderstand in Gleichung (21) eliminiert werden.

In der Literatur ist eine Vielzahl weiterer Verfahren zur Analyse der Atemmechanik beschrieben. Eine Übersicht gibt [14].

Die quantitative Analyse der Atemmechanik des respiratorischen Systems (a) ist die Voraussetzung für das Verständnis der komplexen Wechselwirkung zwischen Patient und Beatmungsgerät, (b) liefert wichtige klinische Information über die Lungenfunktion und den Krankheitsverlauf und (c) ermöglicht es dem Arzt, die Einstellung der Beatmung an die Bedürfnisse des einzelnen Patienten anzupassen.

\section{Literatur}

1. Agostoni E (1972) Mechanics of the pleural space. Physiol Rev 52: 57-128

2. Amato MBP, Barbas CSV, Medeiros DM, Magaldi RB, Schettino, GPP, Filho GL, Kairalla RA, Deheinzelin D, Munoz C, Oliveira R, Takagaki TV, Carvalho CRR (1998) Effect of a protective-ventilation strategy on mortality in the acute respiratory distress syndrome. N Engl J Med 338: 347-354

3. Bertschmann W, Guttmann J, Zeravik J, Eberhard L, Adolph M, Wolff G (1990) Atemzugsweise Bestimmung von Compliance und Resistance am Beatmeten. Intensivmed. 27: 42-47
4. Bertschmann W, Guttmann J, Wolff G (1993) Barotrauma. Annual of Cardiac Surgery 6: 41-51

5. Bohl W (1989) Technische Strömungslehre. Vogel, Würzburg (8. Auff.)

6. Bolder PM, Healy TEJ, Bolder AR, Beatty PCW, Kay B (1986) The extra work of breathing through adult endotracheal tubes. Anesth Analg 65: 853-859

7. Bone RC (1976) Diagnosis of causes for acute respiratory distress by pressurevolume curves. Chest 70: 740-746

8. Bone RC (1983) Monitoring ventilatory mechanics in acute respiratory failure. Respir Care 28: 597-604
9. Brochard L, Roudot-Thoraval F, Roupie E, Delclaux C, Chastre J, Fernandez-Mondéjar E, Clémenti E, Mancebo J, Factor P, Matamis D, Ranieri M, Blanch L, Rodi G, Mentec H, Dreyfuss D, Ferrer M, BrunBuisson $\mathrm{C}$, Tobin $\mathrm{M}$, Lemaire $\mathrm{F}$ and the Multicenter Trial Group on Tidal Volume Reduction in ARDS (1998) Tidal volume reduction for prevention of ventilatorinduced lung injury in acute respiratory distress syndrome. Am J Respir Crit Care Med 158: 1831-1838

10. Don HF, Robson JG (1965) The mechanics of the respiratory system during anesthesia. Anesthesiology 26: 168-178 
11. Dreyfuss D, Saumon G (1992) Barotrauma is volutrauma, but which volume is the one responsible? Intensive Care Med 18: 139141

12. Eberhard L, Guttmann J, Wolff G, Bertschmann W, Minzer A, Kohl HJ, Zeravik J, Adolph M, Eckart J (1992) Intrinsic PEEP monitored in the ventilated ARDS patient using a mathematical method. J Appl Physiol 73: 479-485

13. Guttmann J, Eberhard L, Fabry B, Bertschmann W, Wolff G (1993) Continuous calculation of intratracheal pressure in tracheally intubated patients. Anesthesiology 79: 503-513

14. Guttmann J (1998) Analysis of respiratory mechanics during artificial ventilation. Biomed Tech 43: 107-115

15. Guttmann J, Kessler V, Mols G, Hentschel R, Haberthür C, Geiger K (1999) Calculation of intratracheal pressure in the presence of pediatric endotracheal tubes. Crit Care Med (in press)
16. Mead J, Milic-Emili J (1964) Theory and methodology in respiratory mechanics with glossary of symbols. In: Fenn WO, Rahn H (eds): Handbook of Physiology, section 3/I, Amer Physiol Soc, Washington

17. Milic-Emili J (1989) Pulmonary flow resistance. Lung 167: 141-148

18. Neergaard von K, Wirz K (1927) Über eine Methode zur Messung der Lungenelastizität am lebenden Menschen, insbesondere beim Emphysem. Z Klin Med 105: 35-50

19. Neergaard von K, Wirz K (1927) Die Messung der Strömungswiderstände in den Atemwegen des Menschen, insbesondere bei Asthma und Emphysem. Z Klin Med 105: 51-82

20. Otis $\mathrm{AB}$, McKerrow $\mathrm{CB}$, Bartlett RA, Mead J, Mcllroy MB, Selverstone NJ, Radford Jr. EP (1956) Mechanical factors in distribution pulmonary ventilation. J Appl Physiol 8: 427-443

21. Pedley TJ, Schroter RC, Sudlow MF (1977) Gas flow and mixing in the airways. In: West JB (ed): Bioengineering aspects of the lung. Marcel Dekker, New York Basel, pp 163-265

22. Pepe PE, Marini JJ (1982) Occult positive end-expiratory pressure in mechanically ventilated patients with airflow obstruction. Am Rev Respir Dis 126: 166-170
23. Rohrer F (1915) Der Strömungswiderstand in den menschlichen Atemwegen und der Einfluss der unregelmässigen Verzweigung des Bronchialsystems auf den Atmungsverlauf in verschiedenen Lungenbezirken. Pfluegers Arch Gesamte Physiol Menschen Tiere 162: 225-299

24. Rossi A, Gottfried SB, Zocchi L, Higgs BD, Lennox S, Calverly PMA, Begin P, Grassino A, Milic-Emili J (1985) Measurement of static compliance of the total respiratory system in patients with acute respiratory failure during mechanical ventilation: the effect of intrinsic PEEP. Am Rev Respir Dis 131: 672-677

25. Rossi A, Gottfried SB, Higgs BD, Zocchi L, Grassino A, Milic-Emili J (1985) Respiratory mechanics in mechanically ventilated patients with respiratory failure. J Appl Physiol 58: 1849-1858

26. Wright PE, Marini JJ, Bernard GR (1989) In vitro versus in vivo comparison of endotracheal tube airflow resistance. Am Rev Respir Dis 140: 10-16 